\title{
TECHNICAL ASPECTS FOR THE CREATION OF A MULTI-DIMENSIONAL LAND INFORMATION SYSTEM
}

\author{
Charalabos Ioannidis*, Chryssy Potsiou, Sofia Soile, Styliani Verykokou, George Mourafetis, Nikolaos Doulamis
}

School of Rural and Surveying Engineering, National Technical University of Athens, Greece cioannid@ survey.ntua.gr, chryssy.potsiou@gmail.com, ssoile@survey.ntua.gr, st.verykokou@gmail.com,gmourafetis@yahoo.com, ndoulam@cs.ntua.gr

Commission II, WG II/2

KEY WORDS: 5-dimensional modelling, Level of Detail, Selective modelling, Land Information System, Dense matching, Viewer

\begin{abstract}
:
The complexity of modern urban environments and civil demands for fast, reliable and affordable decision-making requires not only a 3D Land Information System, which tends to replace traditional 2D LIS architectures, but also the need to address the time and scale parameters, that is, the $3 \mathrm{D}$ geometry of buildings in various time instances ( $4^{\text {th }}$ dimension) at various levels of detail (LoDs $-5^{\text {th }}$ dimension). This paper describes and proposes solutions for technical aspects that need to be addressed for the 5D modelling pipeline. Such solutions include the creation of a 3D model, the application of a selective modelling procedure between various time instances and at various LoDs, enriched with cadastral and other spatial data, and a procedural modelling approach for the representation of the inner parts of the buildings. The methodology is based on automatic change detection algorithms for spatialtemporal analysis of the changes that took place in subsequent time periods, using dense image matching and structure from motion algorithms. The selective modelling approach allows a detailed modelling only for the areas where spatial changes are detected. The procedural modelling techniques use programming languages for the textual semantic description of a building; they require the modeller to describe its part-to-whole relationships. Finally, a 5D viewer is developed, in order to tackle existing limitations that accompany the use of global systems, such as the Google Earth or the Google Maps, as visualization software. An application based on the proposed methodology in an urban area is presented and it provides satisfactory results.
\end{abstract}

\section{INTRODUCTION}

Current cadastral data models and Land Information Systems (LISs) use a 2D land-parcel definition. However, 3D cadastral information management systems provide several advantages to the end-users engaged in the land development process, including land registries, surveyors, architects, developers, planners, real estate agents, local governments, and owners' corporations. Digital 3D LIS architectures can provide important information for different aspects of land and property management. Specifically, they represent the spatial extent of ownership boundaries in the third dimension of height where layered and stratified ownerships exist. Furthermore, they facilitate the registration of 3D property rights and support land development processes, including issuing of permit plans in dense urban areas, especially for large scale developments, such as bridges and tunnels that cross above or under other developments. They also provide reliable information for decision makers. Finally, 3D LISs may be utilised as a basic layer to integrate with other information layers, such as 3D city models (CityGML), Building Information Models, transportation and utility networks and land use controls.

However, a land information management system is by its nature dynamic, because several spatial-temporal changes may occur. All this information cannot be modelled by a 3D LIS architecture, emerging the need for a 4D land information management system. In this case, the fourth dimension is the time, meaning that time varying $3 \mathrm{D}$ models need to be created.
The main difficulty of a 4D LIS is that several actors are involved, each having their own diverse requirements and information needs. Therefore, it is argued that even the four dimensions are not adequate for the purposes of land management. Hence, an additional dimension, the scale, should be added to complete the digital counterpart for modern urban management architectures. The advantages of 5D modelling, compared with the $4 \mathrm{D}$ one, is that the first performs a time-scale geometric model representation, instead of a temporal representation of the 3D geometric properties. The introduction of the time and scale dimensions into 3D LISs will enhance data management in various levels of detail (LoDs) without gaps and overlaps in time and space.

Nowadays, 5D modelling is being investigated as a new research paradigm for LISs and especially for cadastre applications. The main bottleneck in applying 5D modelling is its computational complexity and the effort required, which makes it practically impossible to be implemented in real life conditions. This is especially evident in cases of 5D cadastre applications, where several periodic 3D models obtained at different scales need to be incorporated in order to implement a 4D digital model representation.

Previous research in spatial information management separates treatment of 3D geometry from scale and time dimensions. In the last decade, much research has been conducted on 4D cadastre and land administration, focusing on the spatial (3D) and temporal aspects of the real property (van Oosterom et al.,

\footnotetext{
* Corresponding author
} 
2006; Doner et al., 2008; Doner et al., 2011; Doner and Biyik, 2013; Vučić et al., 2014; Seifert et al., 2015; Doulamis et al., 2015). Only a few research papers focus on 5D modelling (van Oosterom et al., 2010; Stoter et al., 2012; Ioannidis et al., 2015). Currently, 5D modelling is implemented by a simple aggregation of 3D digital models at different time instances and scales. Although such an approach is appropriate to experimentally prove the importance of 5D modelling at the lab, it is not suitable to be applied for large-scale application scenarios, where time and cost are critical factors. For this reason, a new framework has to be investigated. This paper introduces a predictive spatial-temporal multi-scale modelling approach, which aims at the selective creation of 5D digital models for use in a 5D multi-purpose LIS.

The rest of the paper is organized as follows. Section 2 outlines the general methodology followed for the creation of $5 \mathrm{D}$ models. Section 3 presents in detail the 3D modelling approach at various LoDs and section 4 discusses the selective modelling approach that is part of the proposed 5D modelling scheme. Results of the discussed methodology are presented both in sections 3 and 4 . In section 5, the in-house developed 5D viewer is presented. Finally, section 6 summarizes the conclusions of the paper.

\section{METHODOLOGY}

In this section, the proposed methodology for the creation of multi-scale 3D models of buildings of various time instances is presented. The 5D modelling framework (Figure 1) relies on a selective (predictive) 3D modelling procedure at different time instances and LoDs. The selective modelling scheme improves the automation and cost-effectiveness of 3D capturing of land parcels through automatic change detection techniques and spatial-temporal analysis. The LoD approach, except for facilitating the efficient visualization of large scenes, provides models of various levels of abstraction to different actors (researchers, engineers, urban planners), according to their requirements.

The input data of the proposed 5D modelling framework consist of overlapping aerial and terrestrial images from various time instances, other spatial and cadastral information. 3D models of buildings of a reference epoch are generated through photogrammetric analysis of airborne and terrestrial images through a procedural modelling technique. The fourth dimension is introduced through the creation of 3D models of buildings in areas that undergo a significant spatial-temporal change at subsequent time instances. According to this selective modelling scheme, regions of insignificant changes remain intact. The fifth dimension is introduced via the creation of multi-temporal models that correspond to different LoDs. The derived 5D models are enriched with additional spatialtemporal properties as well as urban, proprietary and cadastral data.

The proposed methodology has been tested in nine urban blocks in the municipality of Kessariani, located in the eastern part of Athens, Greece. 3D reconstruction is applied in three LoDs at two time instances (1983 and 2010). The initial data consist of (a) a stereo pair of analogue greyscale aerial images of 1983 at a scale of 1:7000; (b) a stereo pair of analogue colour aerial images of 2010 at a scale of 1:6000, both with known interior orientation parameters; (c) 14 GCPs well distributed in the overlapping areas of the stereo pairs, collected via RTK GPS measurements; (d) terrestrial images of the building façades; (e) cadastral information collected from the Greek Cadastre.

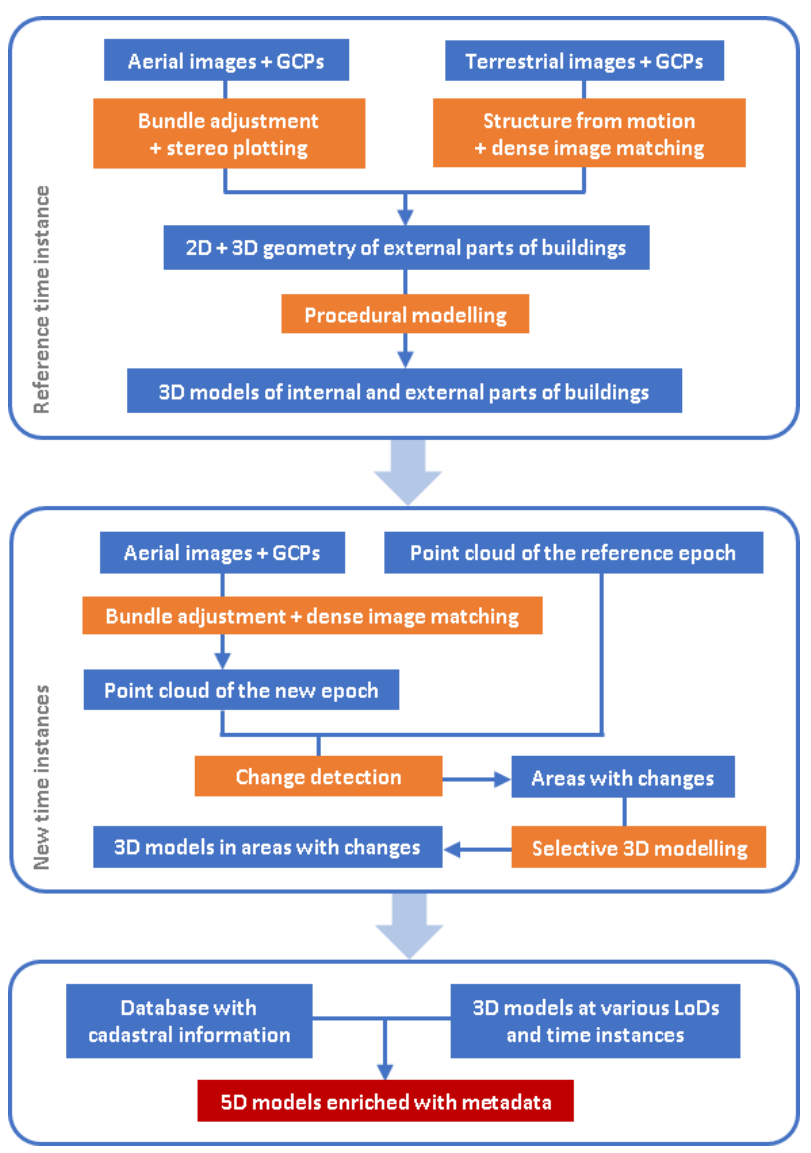

Figure 1. The proposed 5D modelling scheme

\section{3D MODELLING AT VARIOUS LODS}

In this section, the proposed procedure for the creation of $3 \mathrm{D}$ models of buildings at different LoDs is presented. The differentiation of LoDs, as proposed by CityGML may be used. LoD0 is a Digital Surface Mode (DSM) of the region of interest. LoD1 comprises prismatic objects with flat roofs; a building in LoD2 includes information about the roof structures and balconies as well as the wall surfaces; LoD3 denotes architectural models with further detailed wall and roof structures as well as balconies and high-resolution textures are mapped onto these structures (Groger et al., 2012).

\subsection{Image-Based Modelling}

For the production of a high accuracy and quality 3D photogrammetric output using airborne images of the reference epoch, conventional photogrammetric techniques, i.e. bundle adjustment and high precision manual stereo plotting, may be applied. The outputs of this procedure are building outlines and roofs, mass points defining the ground as well as points on the top of man-made constructions. The generation of building footprints is accomplished using the horizontal positions of the outlines of the building roofs, and the corresponding ground elevation, acquired from the digital terrain model (DTM) of the region of interest, created using the stereo plotted ground points. The building footprints along with the outlines of their roofs suffice for the creation of 3D models in LoD1 (Figure 2). 


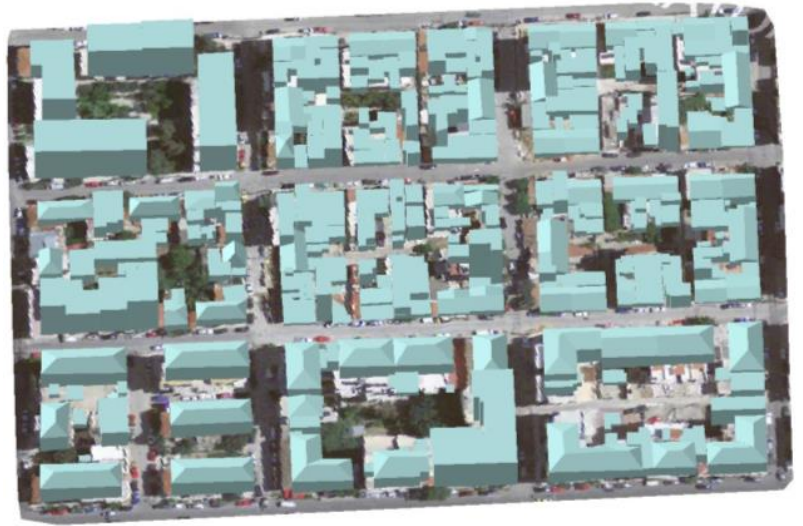

Figure 2. 3D models of external volumes of buildings of the study area in the reference epoch (1983) superimposed on a textured (orthoimage) DTM

For the extraction of detailed 3D building models in LoD2 and LoD3, a combined use of aerial and terrestrial images is necessary (Figure 3). The technological development in the fields of computer vision and digital photogrammetry has provided new tools and automated solutions for 3D modelling. In this context, the most appropriate workflow for the acquisition of $3 \mathrm{D}$ and $2 \mathrm{D}$ photogrammetric products (i.e., point clouds, orthoimages) in cases of terrestrial acquisition of large datasets of images or videos with a variety of orientations and distance from the object, is the application of Structure from Motion (SfM) techniques, followed by Dense Image Matching (DIM) algorithms.

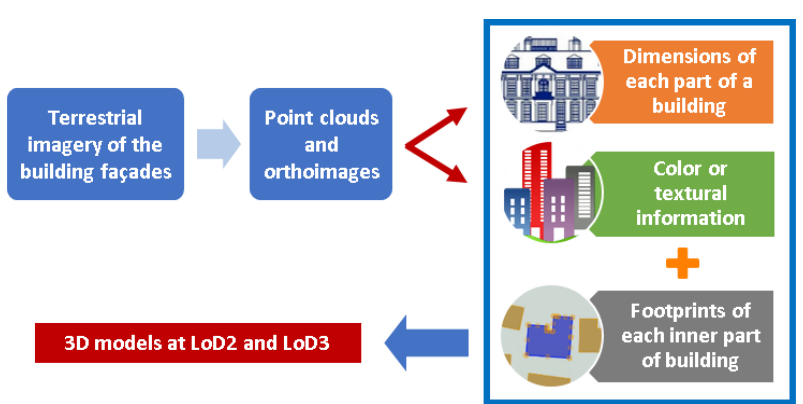

Figure 3. Creation of 3D models at LoD2 and LoD3

SfM methods solve the camera pose and 3D geometry simultaneously using a $2 \mathrm{D}$ image sequence, without the need for approximate camera positions. The extraction of feature points, followed by image matching techniques, the projective reconstruction using a sequential or factorization method, the metric reconstruction via auto-calibration and a bundle adjustment are the steps of a usual processing chain followed by $\mathrm{SfM}$ algorithms. The georeferencing of the SfM sparse point cloud and the camera poses is generally accomplished through the computation of the 3D similarity transformation between the arbitrary coordinate system of the SfM point cloud and the world reference system.

The generation of a dense point cloud is achieved using the results of the SfM procedure through an automated DIM technique, that is, an image pixel-wise matching process that generates a dense Digital Surface Model (DSM) of an area or object. A stereo approach (using a local, global or semi-global method) or a multi-view algorithm may be used. In addition to disparity maps and 3D point clouds, orthoimages may also be obtained, using the results of SfM and DIM procedures.
In this context, the $3 \mathrm{D}$ reconstruction of the sides of every urban block (Figure 4) and the creation of orthoimages (Figure 5) may be accomplished through SfM and DIM techniques using terrestrial images of the building façades and GCPs. The derived point clouds and orthoimages may be used for the extraction of information about the height of floors, the dimensions of doors, windows, balconies and other architectural and construction details. In addition to metric information, textural and colour information, essential for 3D modelling in higher levels of detail, are obtained using the results of SfM and DIM. The existence of obstacles in front of buildings (e.g., parked vehicles, rubbish bins) or on the façades (e.g., awnings in balconies) and the parts of buildings that are not visible from the street level do not allow the full representation of the façades in the images (Figure 5). However, these obstacles do not hinder the subsequent creation of detailed 3D models via procedural modelling, as what is needed from the terrestrial images is only the acquisition of some geometrical elements of the façades and not necessarily the whole representation of them.
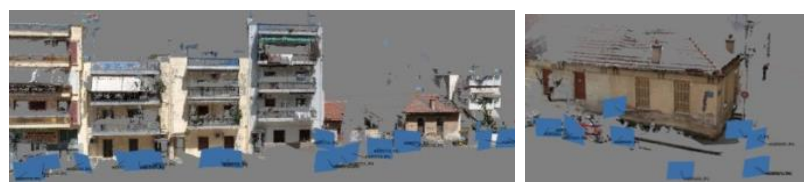

Figure 4. Dense point clouds of sides of an urban block

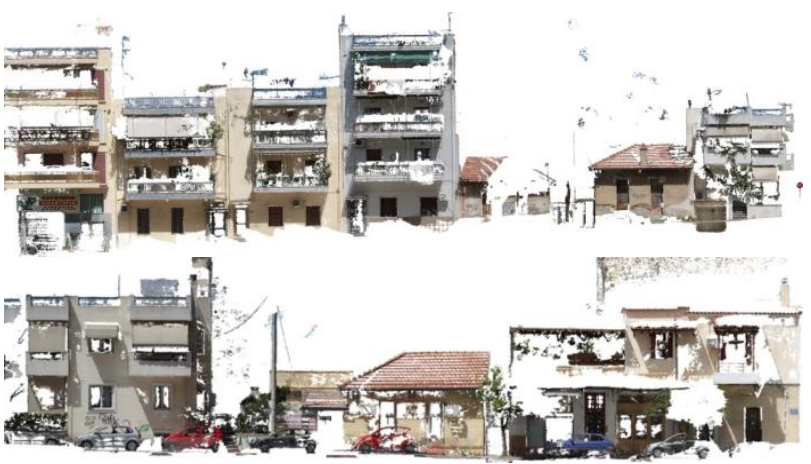

Figure 5. Orthoimages of two sides of an urban block of interest

Instead of the acquisition of terrestrial images of the building façades, low altitude UAV images may also be acquired. Such kind of imagery usually depicts less obstacles and may cover parts of buildings that are not visible from street level. Furthermore, instead of the implementation of DIM for the 3D reconstruction of the sides of urban blocks, terrestrial laser scanning could be used alternatively.

\subsection{Procedural Modelling at Different LoDs}

The 3D model of each building (in LoD1) or each inner part of a building, i.e. apartment, common area, storage area (in LoD2 and LoD3), may be generated through procedural modelling. According to this approach, a model is automatically created through a set of rules, that is, computer code which generates 3D content. Procedural modelling techniques use programming languages for the textual semantic description of a building, which generates a polygonal model (Parish and Müller, 2001; Saldaña, 2015). Furthermore, they require the modeller to critically analyze each structure and describe its part-to-whole relationship using a programming language. An important advantage of procedural modelling is the fact that it allows for the rapid and interactive updating of a model. In addition, the use of attributes and parameters enables the visualization of 
change over time and the representation of different scales, through the introduction of the various LoDs of 3D content (Besuievsky and Patow, 2014; Saldaña, 2015).

Computer Generated Architecture (CGA) shape grammar, as introduced by Müller et al. (2006), may be used for the procedural modelling of buildings. It is a unique programming language specified to generate architectural 3D content. CGA rules are applied to initial shapes to iteratively generate and refine a design by creating more details. The rules defined by CGA grammar enable the procedural creation of complex 3D models of buildings by firstly producing a crude volumetric model, subsequently structuring each façade and finally adding details for doors, windows, balconies, etc., in the desired LoD. The rules are created using metric information, colour and texture for the elements of each building, obtained through the SfM and DIM techniques. They are applied to the footprints of either the buildings (in LoD1) or the inner parts of buildings (in LoD2 and LoD3). In this way, 3D models of various LoDs are created (Figures 6 and 7).

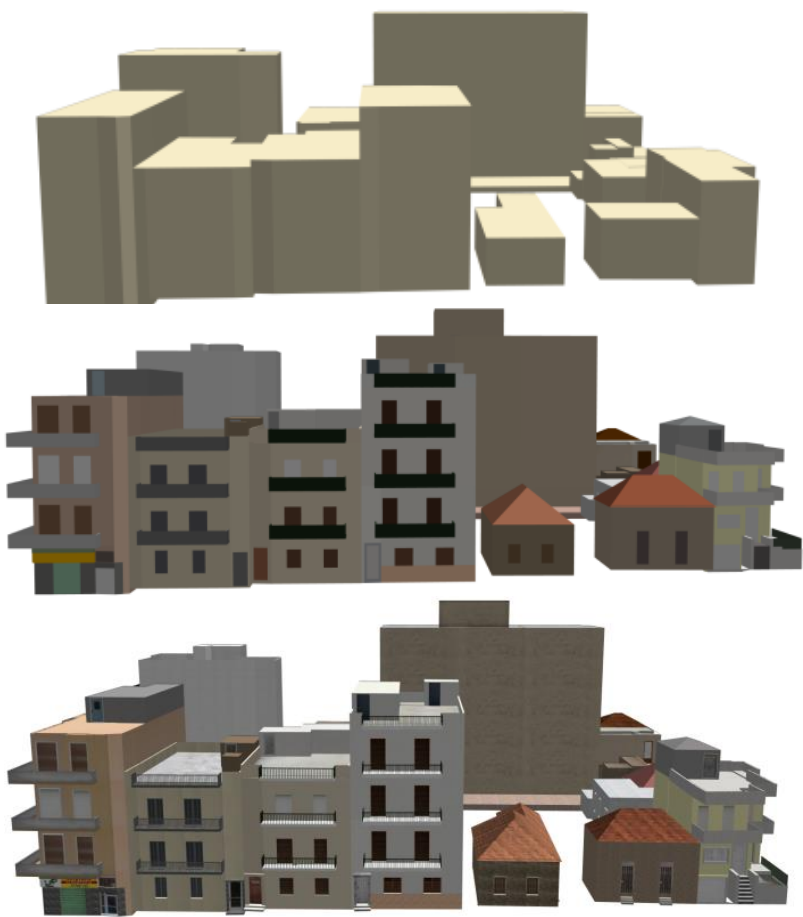

Figure 6. 3D views of the building models of an urban block at LoD1 (top), LoD2 (middle) and LoD3 (bottom)

\section{SELECTIVE MODELLING}

The time dimension in a multi-dimensional LIS is introduced via the creation of $3 \mathrm{D}$ models of buildings at different time instances. It is clear that a time-varying $3 \mathrm{D}$ model should be developed to assess the spatial and temporal diversity of an urban environment. This time-varying 3D modelling results in 4D forms of representations that are necessary for capturing the spatial-temporal changes of the 3D urban environment. Currently, 4D digitization processes are performed under a spatial-temporal independent framework. The main limitation of such an approach is its high computational complexity, making the whole process an arduous and practically impossible task. To address this limitation, the introduction of a novel concept that transforms the time and resource demanding process of $4 \mathrm{D}$ modelling into a cost-effective framework able to be implemented in large scale scenarios was made. The approach, named selective 3D modelling, accelerates the digitization process by exploiting information of $3 \mathrm{D}$ reconstruction from previous time instances.

\subsection{Basic Concept}

A selective 3D modelling scheme is introduced, according to which only regions that undergo a significant spatial-temporal change are 3D modelled at next time instances. For this reason, a spatial temporal analysis has to be applied to indicate the regions of changes, via automated DIM and change detection algorithms. The results of this procedure are change history maps that indicate the regions where spatial-temporal changes have occurred and need to be reconstructed through photogrammetric techniques.

Figure 8 depicts a graphical representation of the selective 3D modelling process, which allows precise reconstruction by exploiting only partial 3D models and appending them onto the 3D information that has been obtained from previous processes. Initially a 3D model is assumed at time instance $t_{k}$ (step 1 of the algorithm). At time instance $t_{k+1}$, an approximate 3D modelling process takes place, exploiting the technology of DIM (step 2). In this way, the respective cost of $3 \mathrm{D}$ reconstruction is significantly decreased with the cost of accuracy. Subsequently, the differences between the precise and the approximate 3D models are computed for the determination of regions of changes (step 3). Spatial filtering algorithms may also be applied to remove isolated noisy 3D points, as a consequence of DIM. In this way, the selective 3D modelling algorithm creates a change history map, which contains spatial 3D information for the regions that have undergone a significant change (step 4). Only the areas of changes undergo precise 3D reconstruction based on the application of photogrammetric methods (step 5). This approach significantly reduces the respective computational and resources demands, since only a small amount of information is precisely $3 \mathrm{D}$ reconstructed. In order to build the final precise model, the non-altered regions of the previous time instances are appended over the current partial precise 3D model (step 6) and the final reconstruction takes place.
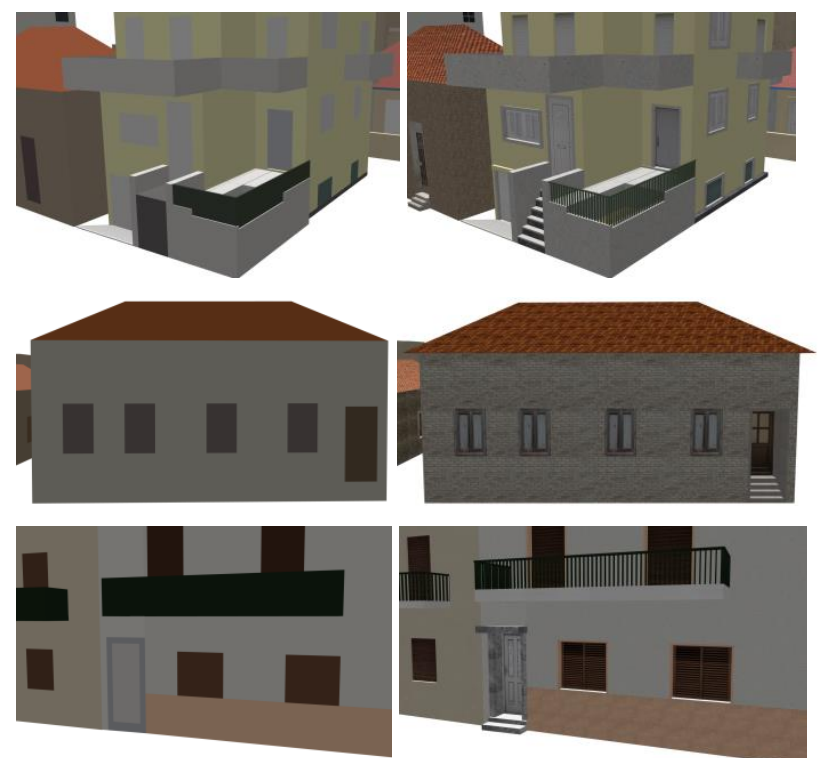

Figure 7. 3D views of the building models of an urban block at LoD2 (left) and LoD3 (right) 


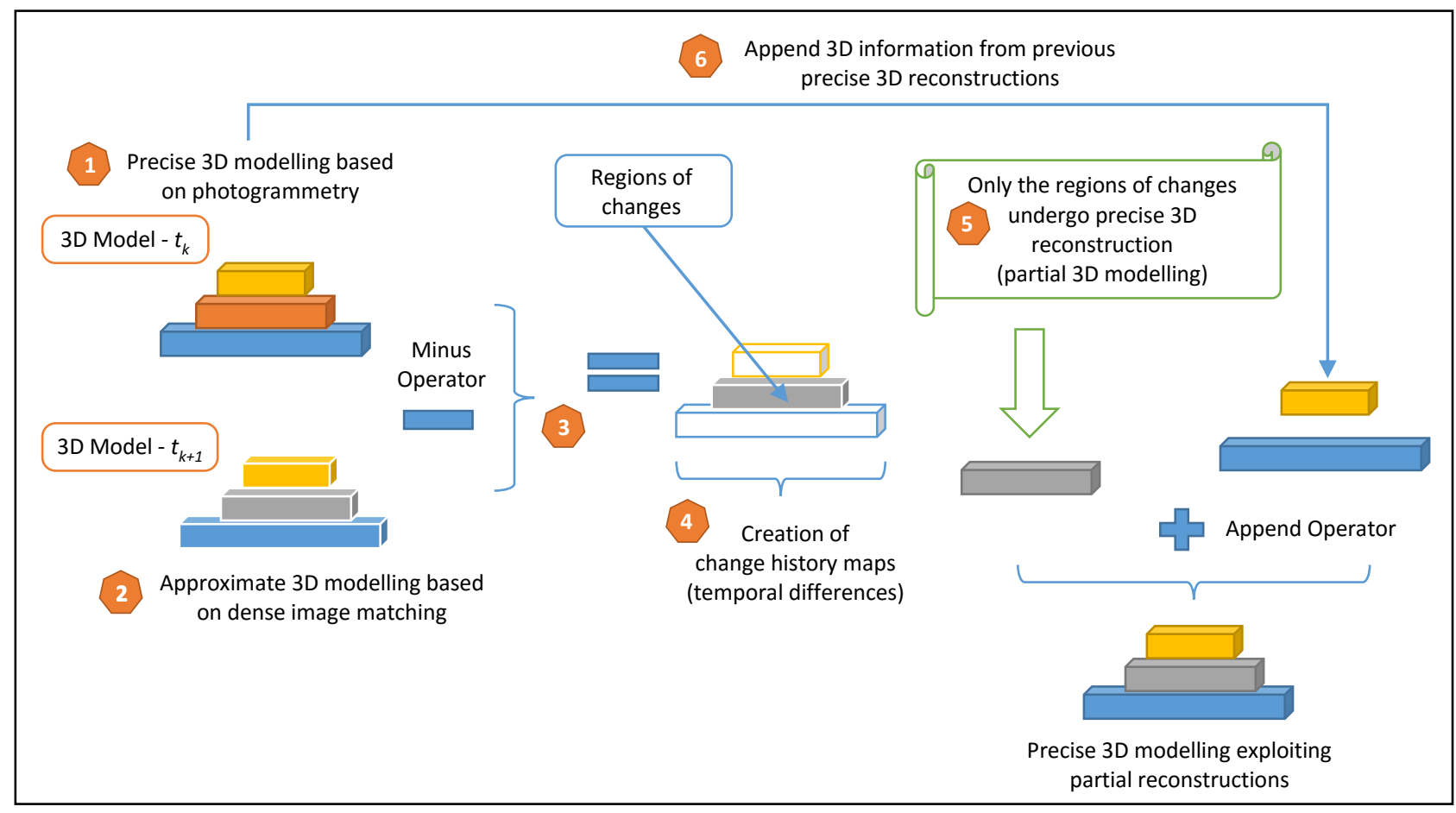

Figure 8. The proposed selective modelling scheme

\subsection{Change Detection}

Much research has been conducted in the fields of photogrammetry and computer vision concerning automatic change detection methods in urban environments (e.g., Ioannidis et al., 2009; Bouziani et al., 2010; Doxani et al., 2010; Stal et al., 2013). The fast and reliable detection of areas with changes (new buildings, additions to old constructions, demolitions, etc.) is a requirement for the creation of $4 \mathrm{D}$ models, that is, $3 \mathrm{D}$ models in various time instances. In land information management applications, where a manual detailed photogrammetric restitution of the areas with changes follows the automated change detection procedure, the accurate determination of the outline of each building that has changed is not necessary; only a rough detection of areas with changes is sufficient.

Specifically, the automated technique of DIM may be applied to the airborne images of a new time instance, after a bundle adjustment procedure, for the fast and cost-effective generation of a 3D point cloud. The latter can then be compared with the manually collected high accuracy point cloud of the reference epoch and the areas with changes can be derived. Some technical issues have to be taken into account in this step. Firstly, the point clouds generally vary significantly in terms of density, due to the different methodologies (i.e., manual and automated techniques) applied for their generation. Thus, they may be transformed into meshes (Figure 9 left and middle) and new point clouds of the same density may be created from the mesh models. Secondly, the application of a smoothing filter (e.g., Laplace) on the meshes of the new epoch, generated through automated techniques, is usually required for the elimination of local outliers due to mismatches from the DIM process. Thirdly, the detection of only significant $3 \mathrm{D}$ changes in the point clouds has to be performed (Figure 9 right). Thus, a thresholding procedure has to be applied; e.g., a threshold of $3.5 \mathrm{~m}$, associated with the height of a typical floor, and a threshold of $2.5 \mathrm{~m}$ above ground, to ignore cases of differences due to vegetation, vehicles, etc. may be implemented. Finally, a post-processing is usually required either by an optical observation and a manual removal of possible false positive changes, or by an automatic process that takes into consideration the shape and size of the entities of interest. In this way, change maps may be obtained, indicating regions that require further $3 \mathrm{D}$ modelling at new time instances.
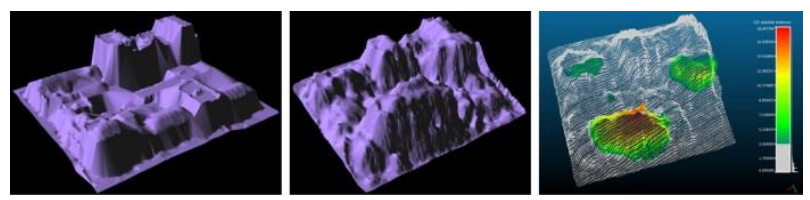

Figure 9. 3D views of the mesh surfaces of an urban block of 1983 (left) and 2010 (middle) created using manual and automatic procedures respectively; detection of significant 3D changes in the urban block (right)

The building change detection results are affected by several parameters, such as the horizontal and vertical uncertainty and the roughness of the point clouds, the interpolation due to occlusions, the performance of the DIM algorithm, the method of the point cloud comparison, etc. The final products of the change detection process, can be superimposed on existing true orthoimages, DSMs, topographic vector data and GIS data.

\subsection{Predictive Modelling at Different Epochs}

Regions which have undergone a significant change at a new time instance are modelled in detail via typical photogrammetric stereo plotting using airborne images in combination with SfM and DIM algorithms, applied in terrestrial imagery. The final creation of their 3D models in various LoDs is accomplished through procedural modelling. The procedure described in section 3 may be used for the modelling of areas with changes in a new epoch. In this way, 3D models of buildings in various LoDs of different time instances may be obtained (Figure 10). 

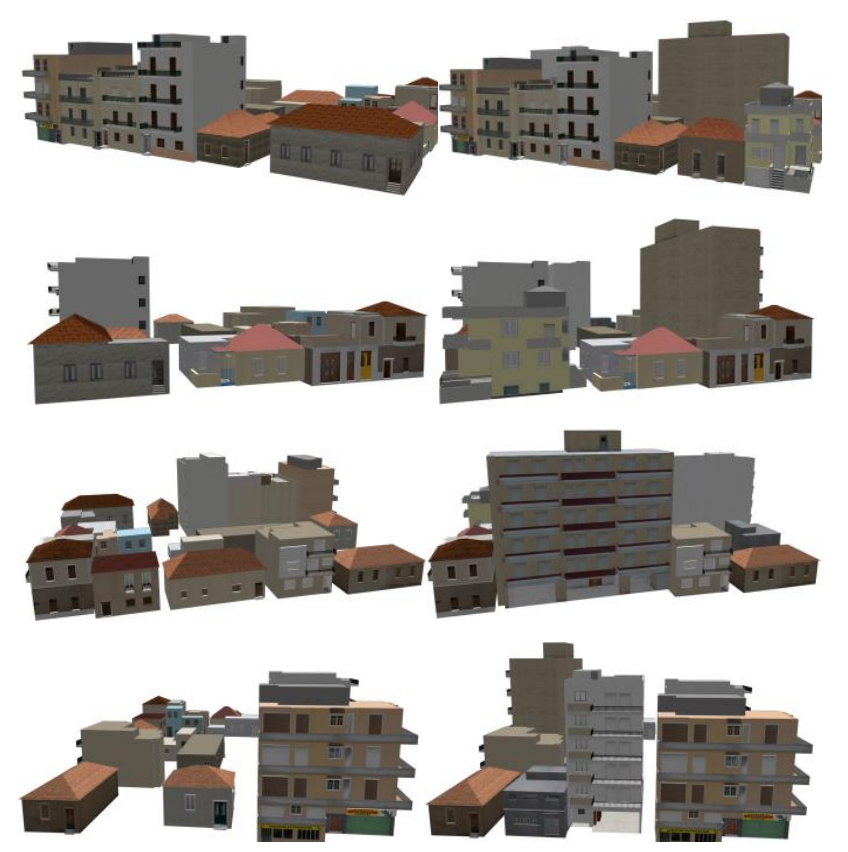

Figure 10. 3D views of the building models of an urban block in the years 1983 (left) and 2010 (right) at LoD3

\section{5D LAND INDOFRMATION SYSTEM}

\subsection{Data Enrichment}

After the creation of the 3D models an external database that can support both spatial and semantic features of buildings is designed and developed. This database is based on the descriptive metadata model of the Hellenic Cadastre and includes three types of data:

- spatial data (geometry and texture); these include the 2.5D footprints, that refer to properties, buildings and land parcels, and models of 3D COLLADA (with textures)

- time (period of validity of property rights and buildings)

- semantic (cadastral data).

The 3D models along with the external database create a 5D Land Information System, which include all data and their relation to cover the needs of various users and applications, such as 2D/3D cadastre, real estate, urban regeneration, queries to examine buildings and parcels within a specific area for urban planning purposes, etc.

Figure 11 shows the database scheme, illustrating the 9 tables that compose it, their fields (columns), the primary keys and their relationships as well as the number of records in each table. The concept supposes that the land is divided into parcels so that each piece of land relates only to one parcel. The parcels are described in the table 'cad_parcel', which holds two unique keys 'parcel_id' and 'kaek'. 'Parcel_id' is a unique identifier with no special meaning while 'kaek' is a code number that consists of information relating to the municipality and wider areas named sectors and sections. Each parcel may have property units with attributes described in table 'cad_prop'. For each property unit there will be registered rights (e.g., percentage of ownership) which are created based on legal documents; these documents are described in table 'cad_doc'. Furthermore, in table 'cad_right_cat_lut' the type of each right is described (e.g., easement) and each right must refer to a beneficiary which is described in table 'cad_ben'. The table 'cad_doc_ben_right' is used to correlate the information between the property unit, the right, the document and the beneficiary. Table 'cad_prop_parcel' relates the property units with the parcels since one parcel may have many property units. Table 'cad_parcel_legal' extends the attributes of each parcel in order to describe legal elements valid for the land parcel (e.g., building to plot ratio, maximum permitted height, etc.), urban planning or architectural data (e.g., land use), real estate values (zone value, tax and commercial values), etc. Finally, each parcel may have a number of buildings with attributes that are described in table 'cad_bldng', such as construction date, number of floors, etc.

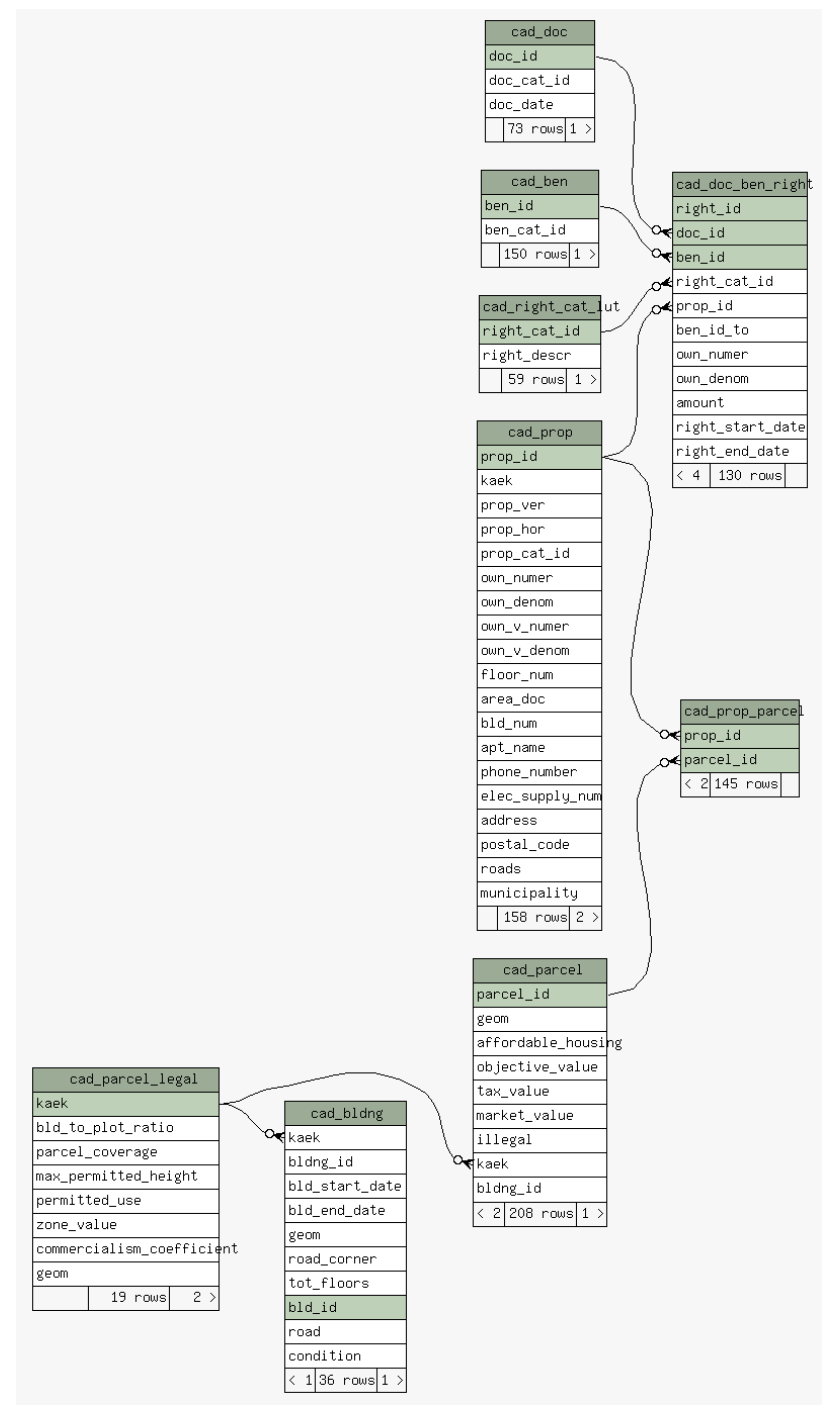

Figure 11. External database scheme including the tables, fields, keys and relationships

\subsection{D viewer (LoP5Dv)}

There already exist many free and commercial viewers for visualizing CityGML models (Nagel et al., 2014), e.g., the Aristoteles viewer developed by the Institute for Cartography and Geoinformation, University of Bonn. In addition, there are various Import/Export tools to various formats for the visualization of the models; e.g., KML files as input to Google Earth.

The KML files that define the planar and vertical location of models of 3D COLLADA (with textures) in Google Earth, due to coordinate systems transformations and cartographic 
background inaccuracy (use of satellite images instead of orthoimages), causes errors in the location of the buildings. To tackle these limitations that accompany the use of global systems, such as the Google Earth or the Google Maps, a new $5 \mathrm{D}$ viewer, named LoP5Dv, was developed. The $5 \mathrm{D}$ viewer is designed to focus on accuracy and customization so that fully tailor made solutions may be implemented, allowing the use of accurate cartographic background (e.g., orthoimages) and right planar and vertical location of buildings.

The LoP5Dv viewer is a custom made software that was developed from scratch on C++ and C\#.NET using OpenGL, without the use of any commercial, open source or other existing API or library. It can read proprietary data that are being converted from other GIS systems, such as ESRI's City Engine; a custom tool to migrate the data from City Engine to LoP5Dv was implemented. The LoP5Dv can be connected to any database and perform SQL queries that can be visualized. The database tier is isolated from the rest of the LoP5Dv so that different database connectors can be implemented. All 3D visualized objects, that exist within the LoP5Dv, are bound to a unique ID that is included in the database and refers to each property. It includes default queries but may also visualize any query that the user can enter in SQL syntax (Figure 12).

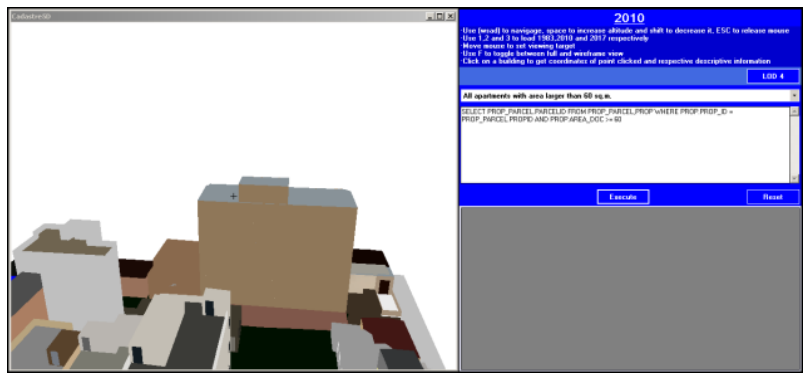

Figure 12. SQL queries
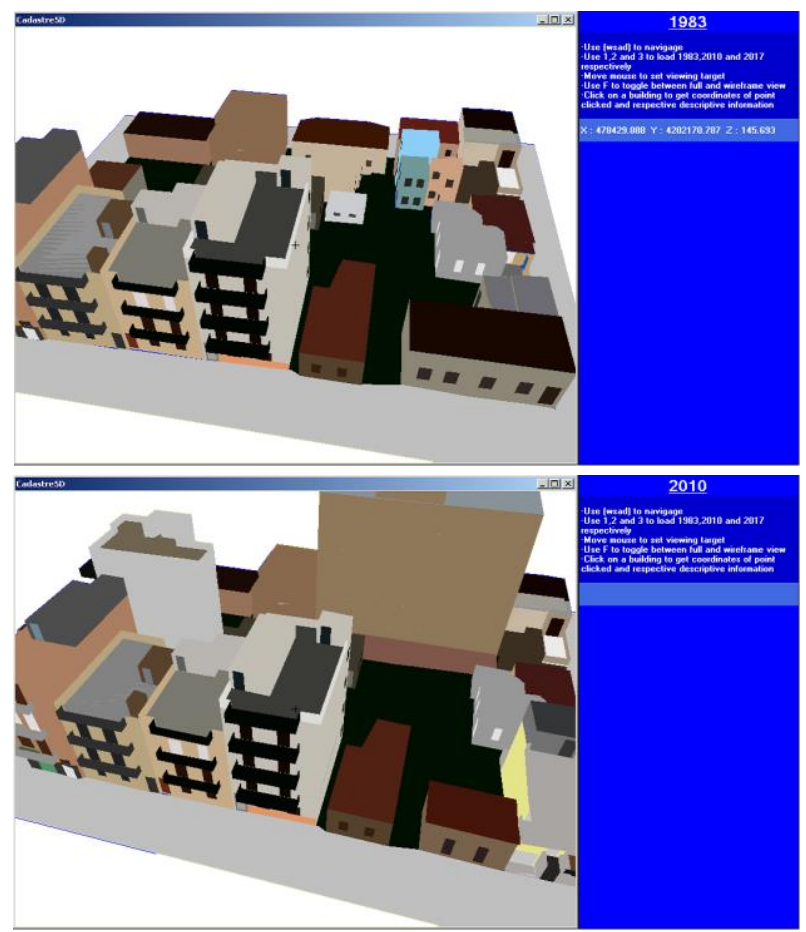

Figure 13. 3D view of the building models at LoD2 in the year 1983 (top) and 2010 (bottom)
The 5D viewer can visualize information based on various criteria including time. It comes with predefined three different time periods in the developed case study (Figure 13).

LoDs are being handled automatically based on the distance of the viewer from the scene. Predefined values have been set in order to provide best viewing experience (Figure 14). The system uses the centre of the scene as a starting point and calculates the distance between the viewer and the centre of the scene. Based on this distance, the system uses various thresholds in order to choose the appropriate LoD. The bigger the distance, the smaller the LoD. After a number of trials, predefined thresholds were selected, but can also be easily changed programmatically.
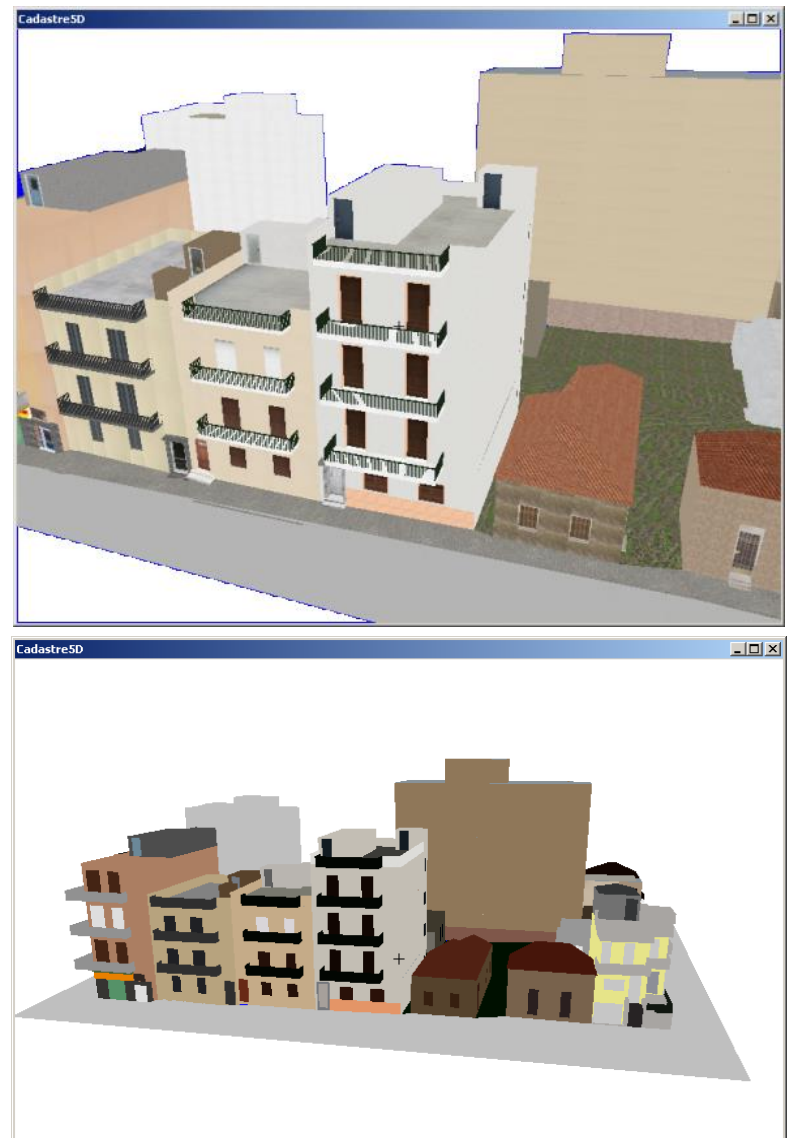

Figure 14. 3D view of the building models of 2010 at LoD3 (top) and LoD2 (bottom)

\section{CONCLUSIONS}

Nowadays, there is a great and urgent global need for smart, reliable, fast, affordable, inclusive and functional solutions, tools and methods that will facilitate the good management of land and good urban governance. In this context, the need for multi-dimensional LISs is evident. The technological development in the fields of computer vision and digital photogrammetry provides new tools and automated solutions for applications in urban studies and cadastre, associated with urban development, 3D modelling, automatic change detection, etc. According to the global needs and current international trends, in this paper a 5D multi-purpose LIS architecture, which handles 3D geometry, time and scale as separate dimensions, is proposed and technical aspects that need to be addressed in the 5D modelling pipeline are discussed. 
Experimental results in an urban area in Greece are outlined and an in-house developed 5D viewer is presented. Such a multidimensional LIS is expected to enable public agencies, the private sector, and citizens to cooperate more efficiently together to develop the strategy, set the regulations and implement land management policies through a fit-for-purpose geospatial infrastructure that will collect, manage and disseminate geospatial data about the ownership, use, development procedures, value of properties and housing aspects.

\section{ACKNOWLEDGEMENTS}

This research was conducted in the framework of the research project "5 Dimensional Multi-Purpose Land Information System" (5DMuPLIS), which is co-funded by the EU (European Regional Development Fund/ERDF) and the General Secretariat for Research and Technology under the framework of the Operational Programme "Competitiveness and Entrepreneurship", "Greece-Israel Bilateral R\&T Cooperation 2013-2015".

\section{REFERENCES}

Besuievsky, G. and Patow, G., 2014. Recent advances on LoD for procedural urban models. In: Proc. of the 2014 Workshop on Processing Large Geospatial Data, Cardiff, UK, Vol. 8.

Bouziani, M., Goïta, K. and He, D.C., 2010. Automatic change detection of buildings in urban environment from very high spatial resolution images using existing geodatabase and prior knowledge. ISPRS Journal of Photogrammetry and Remote Sensing, 65(1), pp. 143-153.

Doner, F. and Biyik, C., 2013. Conformity of LADM for modeling 3D/4D cadastre situations in Turkey. In: Proc. of LADM 2013, Kuala Lumpur, Malaysia.

Doner, F., Thompson, R., Stoter, J., Lemmen, C., Ploeger, H. and van Oosterom, P., 2008. 4D land administration solutions in the context of the spatial information infrastructure. In: Proc. of FIG Working Week 2008, Stockholm, Sweden.

Doner, F., Thompson, R., Stoter, J., Lemmen, C., Ploeger, H., van Oosterom, P. and Zlatanova, S., 2011. Solutions for 4D cadastre - with a case study on utility networks. Int. J. Geogr. Inf. Sci., 25(7), pp. 1173-1189.

Doulamis, A., Soile, S., Doulamis, N., Chrisouli, C., Grammalidis, N., Dimitropoulos, K., Manesis, C., Potsiou, C. and Ioannidis, C., 2015. Selective 4D modelling framework for spatial-temporal land information management system. In: Proc. of SPIE 9535, RSCy2015, 953506, Paphos, Cyprus.

Doxani, G., Karantzalos, K and Tsakiri-Strati, M., 2010. Automatic change detection in urban areas under a scale-space, object-oriented classification framework. In: The International Archives of the Photogrammetry, Remote Sensing and Spatial Information Sciences, Ghent, Belgium, Vol. XXXVIII-4/C7.

Groger, G., Kolbe, T.H., Nagel, C. and Häfele, K.H., 2012. OGC City Geography Markup Language (CityGML) Encoding Standard. Open Geospatial Consortium.

Nagel, C., Benner, J. and Häfele, K.H., 2014. Freeware: Free tools for visualizing and editing CityGML files,
http://www.citygmlwiki.org/index.php/Free_Software (20 Mar. 2016)

Ioannidis, C., Psaltis, C. and Potsiou, C., 2009. Towards a strategy for control of suburban informal buildings through automatic change detection. Computers, environment and urban systems, 33(1), pp. 64-74.

Ioannidis, C., Verykokou, S., Soile, S. and Potsiou, C., 2015. 5D Multi-Purpose Land Information System. In: Proc. of UDMV 2015, Delft, The Netherlands, pp.19-24.

Müller, P., Wonka, P., Haegler, S., Ulmer, A. and van Gool, L., 2006. Procedural modeling of buildings. ACM TOG, 25(3), pp. 614-623.

Parish, Y.I. and Müller, P., 2001. Procedural modeling of cities. In: Proc. of SIGGRAPH '01, Los Angeles, CA, USA, pp. 301308.

Saldaña, M., 2015. An Integrated Approach to the Procedural Modeling of Ancient Cities and Buildings. Digital Scholarship in the Humanities, 30(1), pp. i148-i163.

Seifert, M., Gruber U. and Riecken, J., 2015. Germany on the way to 4D-cadastre. In: Proc. of WCS-CE, Istanbul, Turkey.

Stal, C., Tack, F., De Maeyer, P., De Wulf, A. and Goossens, R., 2013. Airborne photogrammetry and lidar for DSM extraction and 3D change detection over an urban area-a comparative study. International Journal of Remote Sensing, 34(4), pp. 1087-1110.

Stoter, J., Ledoux, H., Meijers, M., Arroyo, Ohori, K. and van Oosterom, P., 2012. 5D modeling - applications and advantages. In: Proc. of 4th Geospatial World Forum, Amsterdam, The Netherlands.

van Oosterom, P., Ploeger, H., Stoter J., Thompson, R. and Lemmen, C, 2006. Aspects of a 4D Cadastre: A First Exploration. In: Proc. of XXIII International FIG Congress, Munich, Germany.

van Oosterom, P. and Stoter, J., 2010. 5D data modelling: full integration of $2 \mathrm{D} / 3 \mathrm{D}$ space, time and scale dimensions. GIScience 2010, LNCS 6292, pp. 310-324.

Vučić, N., Roić, M. and Markovinović, D., 2014. Towards 3D and 4D Cadastre in Croatia. In: Proc. 4th International FIG 3D Cadastre Workshop, Dubai, United Arab Emirates, vol. 2034, pp. 261-280. 